\title{
A Novel Surgical Approach to Traumatic Intracranial Epidural Hematoma
}

\author{
David Shrestha1, Liang Feng ${ }^{2}$ \\ ${ }^{1}$ Department of General Surgery, Western Regional Hospital, Pokhara, Nepal \\ ${ }^{2}$ Department of Neurosurgery, First Affiliated Hospital of Liaoning Medical University, Liaoning, China \\ Email: pokhrelidavid@yahoo.com
}

How to cite this paper: Shrestha, D. and Feng, L. (2017) A Novel Surgical Approach to Traumatic Intracranial Epidural Hematoma. Open Access Library Journal, 4: e3820.

https://doi.org/10.4236/oalib.1103820

Received: July 11, 2017

Accepted: August 5, 2017

Published: August 8, 2017

Copyright $\odot 2017$ by authors and Open Access Library Inc.

This work is licensed under the Creative Commons Attribution International License (CC BY 4.0).

http://creativecommons.org/licenses/by/4.0/

\begin{abstract}
Objective: Traumatic intracranial epidural hematoma is considered to be the serious complication of head injury requiring appropriate evaluation and surgical intervention. Although craniotomy provides an effective evacuation of the hematoma, there is an insufficient data to support one particular surgical treatment method. The objective of this study is to address the effectiveness of Urokinase instillation via single burr hole in different time interval. Method: Forty two patients with traumatic epidural hematoma, ranging between 15 and 71 years who meet the inclusion criteria were selected for the retrospective study. All the patients were surgically treated in the First Affiliated Hospital of Liaoning Medical University from January 2013 to June 2015. Result: The common complication encountered in this study was headache, dizziness, fever and re-bleeding. In Group-A, 4 (19\%) patients complained of headache, $5(23.8 \%)$ dizziness, $1(4.7 \%)$ fever and re-bleeding occurred in $2(9.5 \%)$ patients. In Group-B, 2 (9.5\%) patients complained of headache, 1 (4.7\%) dizziness, $1(4.7 \%)$ fever and 1 (4.7\%) re-bleeding. Out of forty two patients, 3 (7.1\%) patients encountered re-bleeding and received craniotomy. In this study, Group B demonstrates better outcome than Group A $(\mathrm{P}<0.028)$. Conclusion: Single burr hole with Urokinase instillation is safe, feasible and effective technique in the treatment of traumatic epidural hematoma in selected cases where close regular clinical and radiological monitoring is possible.
\end{abstract}

\section{Subject Areas}

Neurology, Surgery \& Surgical Specialties

\section{Keywords}

Urokinase, Single Burr Hole, Traumatic Epidural Hematoma, Hematoma Liquefaction 


\section{Introduction}

Epidural hematoma (EDH) is a potentially serious but treatable sequel of head injury. The incidence of EDH in the population with traumatic brain injury (TBI) has been reported to be in the range of 3\% - 4\% [1] [2] [3] [4] and the peak prevalence of EDH is in the second decade of life. EDH is usually unilateral in more than $95 \%$ of cases, however bilateral or multiple are also reported which account for $2 \%-5 \%$ of all acute epidural hematomas in adults [1] [2] [5] [6].

Computed Tomography (CT) scan has been adopted as primary choice of investigation in the evaluation of suspected intracranial epidural hematoma [7] [8]. The preoperative radiological parameters, such as hematoma volume, degree of midline shift, presence of additional intracranial lesions and neurological status of the patient, have been reported to influence the outcome of EDH [6] [9]. Patients with EDH who meet surgical criteria and receive surgical treatment can have an excellent prognosis presumably owing to limited underlying primary brain damage [10]. Although craniotomy provides more complete evacuation of the epidural hematoma, there are insufficient data to support one particular surgical treatment method [1] [11]. Recently minimal invasive technique has been attempted and valuable outcomes were reported in various literature. It is reported that burr hole evacuation followed by drainage under negative pressure is a safe and effective method for emergency management of pure traumatic epidural hematoma [12]. Furthermore, the twist drill trephination and installation of Urokinase has been described as useful in selective patients without any casualties [13] [14]. It is demonstrated that the use of Urokinase promotes clot lysis and resorption without producing neurotoxicity, histopathological alterations or recurrent bleeding [8]. The fibrinolysis technique has emerged as the most promising surgical modality that has been proved to be safe and effective treatment option for lysis of subarachnoid blood, intraventricular hemorrhage, intracerebral hemorrhage [8] [15] [16] [17] and traumatic epidural hematoma [13] [14]. This study presents novel method of evacuating traumatic EDHs using single burr hole with Urokinase instillation in different time intervals.

\section{Materials and Methods}

Forty-two patients $(n=42)$ range between 15 to 71 years old (mean age $34 \pm$ 16.30) were diagnosed as traumatic epidural hematoma with CT scan. All patients were surgically treated in the First Affiliated Hospital of Liaoning Medical University from January 2013 to June 2015. Among these population 33 were male and 9 were female. Road traffic accident was the major cause of trauma followed by physical assaults. The Glasgow Coma Scale (GCS) score were 8 to 15 during admission. All the medical records and case materials were retrospectively reviewed. Coagulation profile such as Hematocrit, Platelet count, activated Partial Thromboplastin Time (aPTT), Prothrombin Time and International Normalized Ratio (PT/INR), Bleeding Time(BT) and Clotting Time(CT) was 
also reviewed which were within normal range. CT scans were analyzed; hematoma location, size and associated mass effect and parenchymal injury were also carefully assessed.

Inclusion criteria: GCS $\geq 8$, Clear history of trauma, Single hematoma lesion, Supratentorial hematoma, Midline shift $\leq 1 \mathrm{~cm}$ and Informed consent from patient.

Exclusion criteria: GCS < 8, Patient with Polytrauma, Coagulation disorder/ coagulopathy, Depressed skull fracture, and Previous history of stroke; intracranial aneurysm; AV malformation.

Informed consent was taken from the patients who met the inclusion criteria. Patients were allocated into two groups: Group A-surgery within 3 - 5 days of onset and Group B-surgery beyond day 5. Patients were categorized according to their general condition, stability, neurological status and CT findings: blood volume, thickness and midline shift during admission (Table 1). The preferred surgery day was selected according to pathophysiology of hematoma liquefaction [18]. According to Hideo et al., epidural hematoma begins degenerative changes in 3 to 5 day of onset. Within 6 to 10 days blood often become partially clotted and crumbly and usually liquefies at 25 - 30 days [18].

\subsection{Treatment Method}

All patients received intravenous antibiotics on the day of surgery followed by four consecutive days. Patients were shifted to the operation room. Under local anesthesia, single burr hole was drilled over the largest hematoma area according to CT scan scale. Hematoma was allowed to flow out itself and visible clot was removed to create some space for the drain and to instill Urokinase without fur-

Table 1. Computed tomography findings and group division of traumatic epidural hematoma.

\begin{tabular}{|c|c|c|c|c|c|}
\hline Details & $\begin{array}{l}\text { Total } \\
\mathrm{n}=42\end{array}$ & Mean \pm SD & $\begin{array}{c}\text { Group A } \\
\begin{array}{c}(3-5 \text { day) } \\
n=21\end{array}\end{array}$ & $\begin{array}{c}\text { Group B } \\
\text { (>5 day) } \\
n=21\end{array}$ & $P$ value \\
\hline \multicolumn{6}{|l|}{ Volume: } \\
\hline$<30 \mathrm{ml}$ & 8 & $27.12 \pm 3.13$ & 0 & 8 & \\
\hline $31-40 \mathrm{ml}$ & 19 & $34.52 \pm 2.65$ & 11 & 8 & $\mathrm{X}^{2}=10.1404$ \\
\hline $41-60 \mathrm{ml}$ & 15 & $49.66 \pm 5.63$ & 10 & 5 & $\mathrm{P}=0.006281$ \\
\hline \multicolumn{6}{|l|}{ Thickness: } \\
\hline $10-20 \mathrm{~mm}$ & 10 & $14.60 \pm 3.47$ & 0 & 10 & \\
\hline $21-30 \mathrm{~mm}$ & 18 & $24.83 \pm 3.03$ & 7 & 11 & $\mathrm{X}^{2}=24.8889$ \\
\hline $31-40 \mathrm{~mm}$ & 14 & $34.85 \pm 3.48$ & 14 & 0 & $\mathrm{P}=0.00001$ \\
\hline \multicolumn{6}{|l|}{ Midline shift: } \\
\hline No shift & 7 & & 0 & 7 & \\
\hline $0-5 \mathrm{~mm}$ & 18 & $3.5 \pm 0.98$ & 7 & 11 & $X^{2}=15.0065$ \\
\hline $6-10 \mathrm{~mm}$ & 17 & $7.47 \pm 1.28$ & 14 & 3 & $\mathrm{P}=0.000551$ \\
\hline
\end{tabular}


ther increase in intracranial pressure. Draining tube was connected to compressible drain bulb with 3-way stopcock. The minimal negative pressure created by the compressing bulb helps the liquefied hematoma to drain easily. After an hour of surgery, no drainage of fresh blood was confirmed and 30,000 - 50,000 IU of Urokinase dissolved in $3-4 \mathrm{ml}$ of normal saline was injected into the epidural cavity via 3-way stopcock. Another $3 \mathrm{ml}$ normal saline was pushed to flush the tube. Drainage tube was occluded for 2 - 3 hours based on clinical manifestation of the patient and then released. During this time period patients were advised to change the head position often to spread the injected Urokinase all over the cavity and allow early dissolution of clots. The Urokinase was used twice a day for 2 - 3 days based on the patient tolerance and the CT findings. According to the changes in patient clinical manifestation and daily collection in the drain CT scan was performed every 24 hours to check the left over hematoma in epidural space. The drainage tube was not removed until all the clots were resolved as suggested by CT scans. Urgent studies were performed if an acute neurological deterioration was recognized.

\subsection{Static Analysis}

Statistical analysis was conducted using SPSS 20.0 software. The outcome between two groups is analyzed using Pearson's Chi square test. P values $<0.05$ were considered to indicate statistical significance.

\section{Results}

In this study time for detecting hematoma after trauma was 2 - 5 hours (mean $3.14 \pm 1.26$ hour). The mean time of surgery in group- $A$ was $3.57 \pm 0.74$ day and in group-B $6.19 \pm 0.60$ day. Of all 42 included cases, site of hematoma located in temporal region were 23 (54.7\%), 12 (28.5\%) in frontal, 5 (11.9\%) in temporoparietal and $2(4.7 \%)$ in occipital region (Table 2). The common complication encountered following the use of Urokinase was headache, dizziness, fever and re-bleeding (Table 3). In Group-A, 4 (19\%) patients complained of headache, 5 (23.8\%) dizziness, 1 (4.7\%) fever, and re-bleeding occurred in $2(9.5 \%)$ patients in which one had on first day and another on third day of use of Urokinase. In Group-B, 2 (9.5\%) patients complain of headache, 1 (4.7\%) dizziness, 1 (4.7\%) fever and re-bleeding in $1(4.7 \%)$ patient on the first day of Urokinase use.

The nature of headache was mild and the fever was low grade. Fever was treated with intravenous Dexamethason whereas headache was alleviated by release of occluded drain. It is believe that when fibrinolytic activity takes place the expansion phase of the clot occurs causing some degree of fluctuation in intracranial volume and pressure, resulting headache [19]. Patients with re-bleeding complained of severe pulsatile headache and gradually neurological deterioration occurred leading to immediate conventional craniotomy. At once patient was shifted to Operation Theater. Under general anesthesia craniotomy was performed. The volume of hematoma was near double compare to prior urokinase use. Patient from the group A who re-bleed on third day of urokinase use 
Table 2. Site of Extra Dural hematoma.

\begin{tabular}{cc}
\hline Site of hematoma & No. of patient $(\mathrm{n}=42)$ \\
\hline Temporal & $\mathrm{n}=23(54.7 \%)($ Right 10; Left 13) \\
Frontal & $\mathrm{n}=12(28.5 \%)($ Right 4 ; Left 8$)$ \\
Temporo-parietal & $\mathrm{n}=5(11.9 \%)($ Right 2 ; Left 3$)$ \\
Occipital & $\mathrm{n}=2(4.7 \%)($ Right 1 ; Left 1$)$ \\
\hline
\end{tabular}

Table 3. Complication of the treatment.

\begin{tabular}{|c|c|c|c|c|c|c|}
\hline \multirow{2}{*}{ Group } & \multicolumn{3}{|c|}{ Complication } & \multirow[b]{2}{*}{ Re-bleeding } & \multirow{2}{*}{ No complication } & \multirow{2}{*}{ Total } \\
\hline & Headache & Dizziness & Fever & & & \\
\hline Group A & $\mathrm{n}=4(19 \%)$ & $\mathrm{n}=5(23.8 \%)$ & $\mathrm{n}=1(4.7 \%)$ & $\mathrm{n}=2(9.5 \%)$ & $\mathrm{n}=9(42.8 \%)$ & $\mathrm{n}=21$ \\
\hline Group B & $\mathrm{n}=2(9.5 \%)$ & $\mathrm{n}=1(4.7 \%)$ & $\mathrm{n}=1(4.7 \%)$ & $\mathrm{n}=1(4.7 \%)$ & $\mathrm{n}=16(76.1 \%)$ & $\mathrm{n}=21$ \\
\hline
\end{tabular}

was tolerating urokinase for two day. Neurological status was improving but on the third day patient suddenly deteriorated. Urgent CT scan showed large hematoma with midline shift. To evaluate the change in hematoma volume CT scan was carried out every 24 hours after the use of Urokinase. In this study Urokinase was used for minimum 2 days to maximum 3 days until CT scan revealed the resolution of the hematoma, then the drainage tube was removed. All the patients recovered uneventfully and were discharged on $4-6^{\text {th }}$ postoperative day except for three patients who underwent craniotomy and were discharged after two weeks (mean hospital stay, day $5.57 \pm 1.61$ ) (Figure 1 and Figure 2).

\section{Discussion}

The pathophysiology of epidural hematoma is that the sequences of cellular response pace exist between different individuals. According to Hideo et al., the first sprouting of fibroblasts from the outer layer of the dura into the epidural blood clot occurs after 2 to 3 days and gradually the fibroblast layer becomes slightly thicken by few cell layers which begins to enter the clot after 3 to 5 days. Then few erythrocytes may demonstrate degenerative changes. Within 6 to 10 days blood often become partially clotted, crumbly and develops a dark brown color. When hematoma reaches to 25 - 30 days blood usually liquefies [18]. During this process solid hematoma liquefie and begin to detach from one particular point or from the area of injured portion. The natural process of transforming the clot facilitates to remove the epidural hematoma effortlessly using Urokinase.

Urokinase is a strong plasminogen activator which specifically cleaves proenzyme/zymogen plasminogen to form active enzyme plasmin and active plasmin is able to break down the fibrin polymers of blood clots [20] [21] [22]. Before the commencement of thrombolytic therapy obtaining coagulation profile is essential and need to be within the normal range. Patient who exhibit reactions 

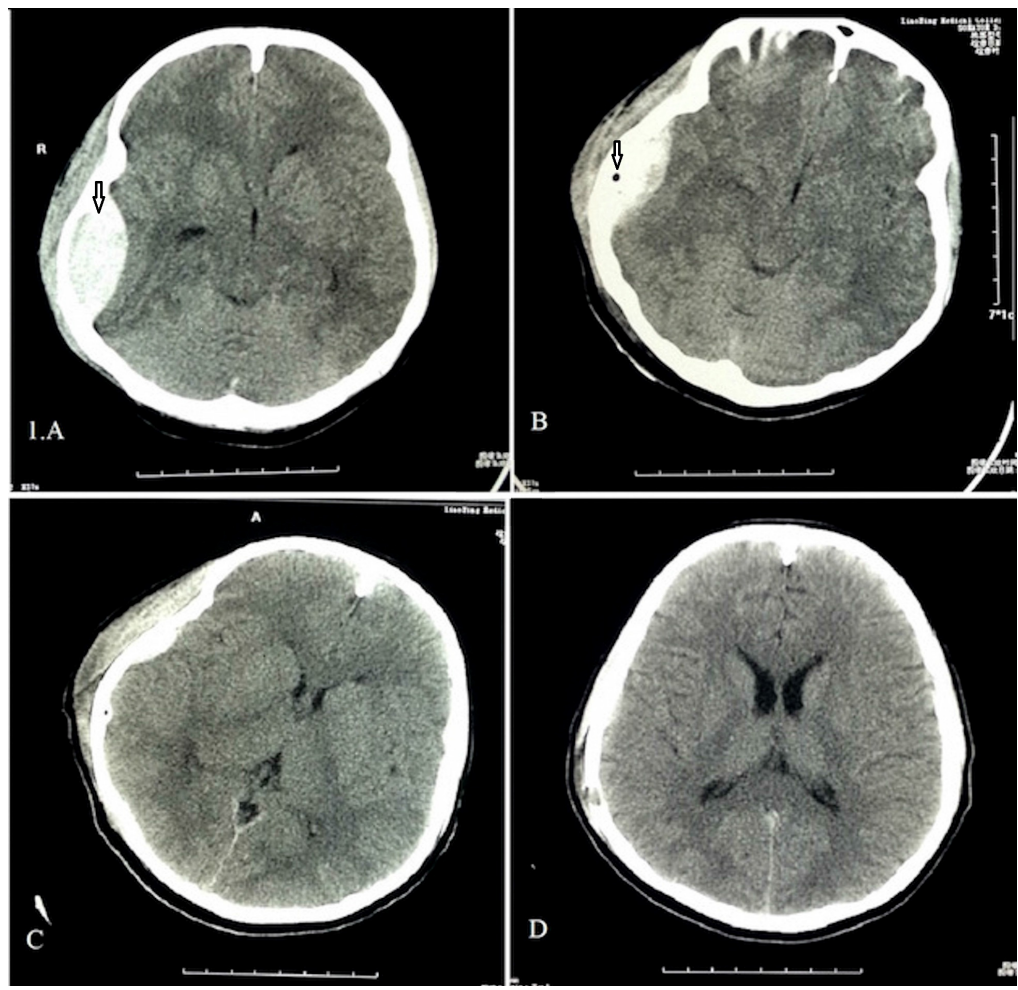

Figure 1. Axial view of Head CT. (A) EDH in right temporal region prior to surgery; (B) $2^{\text {nd }}$ postoperative day with tube in situ; (C) $3^{\text {rd }}$ postoperative day with tube in situ; (D) $5^{\text {th }}$ postoperative day prior to discharge.

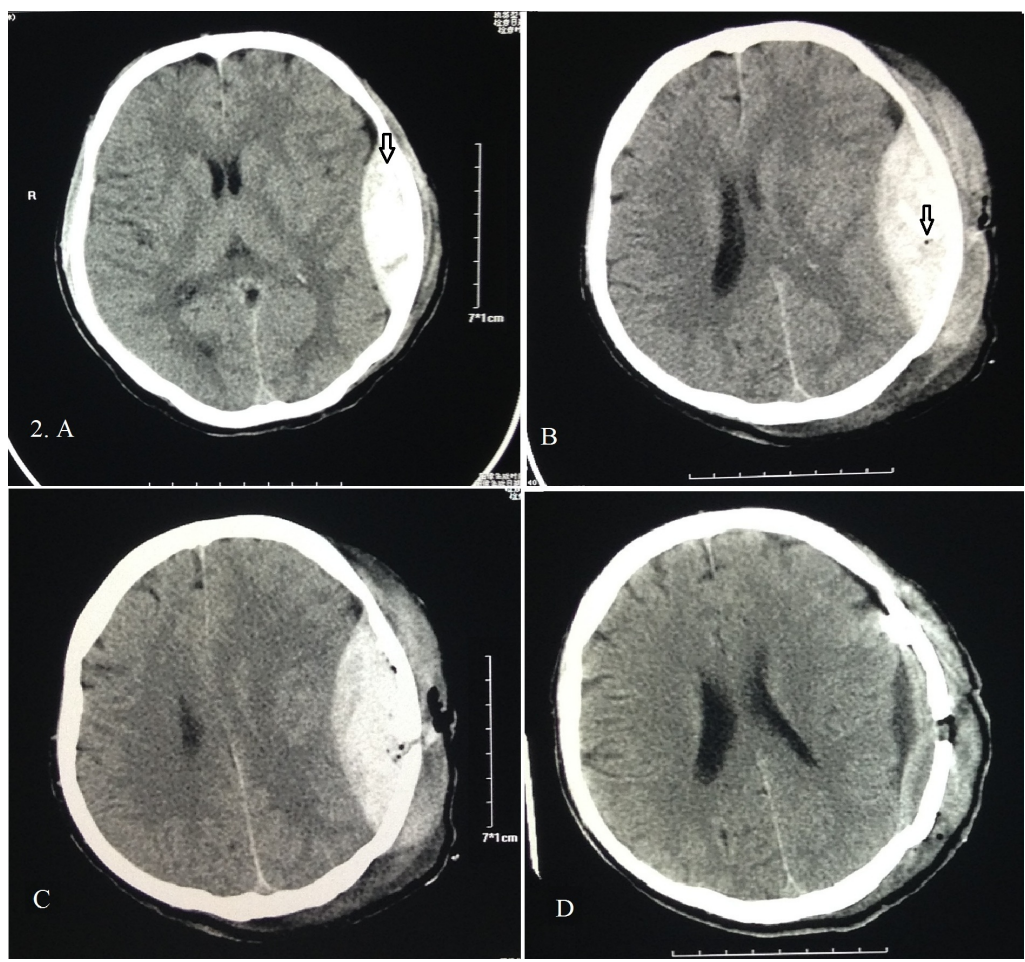

Figure 2. Axial view of Head CT. (A) EDH in left temporal region prior to surgery; (B) \& (C) $1^{\text {st }}$ postoperative scan with tube in situ where size of hematoma increases after instillation of urokinase with mass effect; (D) Post craniotomy scan. 
should be refrained from the treatment, closely monitor and appropriate therapy must be use. Antipyretics such as aspirin and other non-steroidal anti-inflammatory agents which inhibit platelet function may increase the risk of bleeding and should not be used for the treatment of fever during Urokinase use [21] [22]. Following demonstration of clot lysis effect without producing neurotoxicity, histopathological alterations or recurrent bleeding in an animal model by Narayan et al. [8] [23], fibrinolysis technique has emerged as the most promising surgical modality. It has been proved safe and effective treatment option for subarachnoid hemorrhage, intraventricular hemorrhage, intracerebral hemorrhage [8] [15] [16] [17] and traumatic epidural hematoma [13] [14] [24].

The clinical presentation of epidural hematoma somewhat is variable with the mechanism of injury or the site of lesion. Once the diagnosis of epidural hematoma has been made surgical evacuation is usually the standard treatment procedure to prevent mortality or neurologic morbidity. Not all cases of acute EDH require immediate surgical evacuation [11] [25]. If the lesion is too small and patient is in good neurological condition observing patient with frequent neurological examinations is reasonable. Recently non-surgical management of small EDH is increasingly accepted with lesions not associated with mass effect or midline shift [26] [27]. Most of the small to moderate EDHs resolves spontaneously in few weeks to few months [12] [28]. Bullock et al. reported that the resolution of hematoma took 3 - 15 weeks in the conservative management [12]. During the conservative management the reabsorption phase is always accompanied with expansive phase of clot due to fibrinolytic activity and in some cases latter phase may be predominant [19]. The major drawback of conservative treatment is prolong inpatient close monitoring and always remains high chance of re-bleeding.

The decision to perform surgery in patient with traumatic extradural hematoma is depend on neurosurgical judgment. Hematomas volume, secondary mass effect and clinical judgment have been commonly accepted as major criteria for surgery [1]. Patients with EDH who meet surgical criteria and receive surgical treatment can have an excellent prognosis presumably owing to limited underlying primary brain damage [10]. Although craniotomy provides an effective evacuation of hematoma there is insufficient data to support one particular surgical treatment method [1] [11]. A small number of minimally invasive methods have been reported in the literature for the management of EDH in certain circumstances and satisfactory outcome was reported. Noguchi et al., 2010, reported that neonatal EDH is more liquefied than adult counterpart and is amenable to needle aspiration under transcranial ultrasound guidance for massive EDH seen following vacuum-assisted delivery [29]. Also, aspirating cephalhematoma to evacuate communicating epidural hematoma in a newborn infant with no neurological signs has been successfully employed to avoid more invasive surgical intervention by Smets and Vanhauwaert, 2010 [30]. In a clinical series reported by Liu J.T. et al., 2006, 11 of 13 patients harboring traumatic EDH were successfully treated by placement of flexible tube through burr hole fol- 
lowed by continuous suction under negative pressure, and only two cases required secondary craniotomy [31]. The novel method of Urokinase instillation using closed suction drain has been proposed for post-craniotomy epidural hematoma as a feasible method with no complication and better outcome by Park et al., 2008 [14]. And drilling the skull with instillation of Urokinase into hematoma space has been reported in 22 selected cases of traumatic EDH without complication by Liu W. et al., 2008 [13].

Beside some limitation instillation of Urokinase is effective in selective cases. This study may not be favorable in acute stage of traumatic epidural hematoma due to the higher chances of rebleeding that might aggravate the condition. The tamponade effect of blood clot play certain role to stop bleeding during trauma [32]. Therefore early exposure of Urokinase may dissolve the tamponade of the injured vessels sustaining the risk of re-bleeding. Some authors have suggested that by the time of presentation EDH is relatively stable lesion having attained maximum size within minutes of injury [12] [33] [34] [35]. Borovich et al. demonstrated progression of epidural hematoma in $9 \%$ of patients during the first 24 hours [36]. Thus repeated CT scan should be done at least $\sim 6$ to 12 hours of injury followed by every 24 hours. The maximum volume, thickness and midline shift operated in this study was $60 \mathrm{ml}, 40 \mathrm{~mm}$ and $10 \mathrm{~mm}$ respectively. Therefore only hematoma volume is not always the primary indication for early craniotomy. On the basis of our experience we should pay attention to the Urokinase use although there is no overwhelming consensus on the dose. It should be administered gradually in the reduction dose correlating with CT finding to avoid recurrence of bleeding. Strict sterilization procedures need to be maintained. No any neurological deficit or mortality was found throughout the treatment and a year of regular follow up.

\section{Conclusions}

This study demonstrated that single burr hole with Urokinase instillation is safe, feasible and effective technique in the treatment of traumatic intracranial epidural hematoma in selected cases, especially, for the symptomatic patient with GCS greater than eight without signs of cerebral herniation, where close regular clinical and radiological monitoring is accessible. The use of Urokinase is feasible in both groups, however Group B has better outcome than Group A and is statistically significant $(\mathrm{P}<0.028)$.

\section{Consent}

Written informed consents were obtained from the patients for publication of this article and any accompanying images.

\section{Disclosure}

The authors have no proprietary or financial interest in any materials discussed in this work. 


\section{References}

[1] Bullock, M.R., Chesnut, R., Ghajar, J., Gordon, D., Hartl, R., Newell, D.W., et al. (2006) Surgical Management of Acute Epidural Hematomas. Neurosurgery, 58, S7S15.

[2] Gupta, S.K., Tandon, S.C., Mohanty, S., Asthana, S. and Sharma, S. (1992) Bilateral Traumatic Extradural Hematomas: Report of 12 Cases with a Review of the Literature. Clinical Neurology and Neurosurgery, 94, 127-131.

[3] Maggi, G., Aliberti, F., Petrone, G. and Ruggiero, C. (1998) Extradural Hematomas in Children. Journal of Neurosurgical Sciences, 42, 95-99.

[4] Wu, J.J., Hsu, C.C., Liao, S.Y. and Wong, Y.K. (1999) Surgical Outcome of Traumatic Intracranial Hematoma at a Regional Hospital in Taiwan. The Journal of Trauma, 47, 39-43. https://doi.org/10.1097/00005373-199907000-00009

[5] Winn, H.R. (2011) Youmans Neurological Surgery. Section XI-Trauma. 6th Edition, Elsevier, Amsterdam, 3267-3534.

[6] Lee, E.J., Hung, Y.C., Wang, L.C., Chung, K.C. and Chen, H.H. (1998) Factors Influencing the Functional Outcome of Patients with Acute Epidural Hematomas: Analysis of 200 Patients Undergoing Surgery. The Journal of Trauma, 45, 946-952. https://doi.org/10.1097/00005373-199811000-00017

[7] Hamilton, M. and Wallace, C. (1992) Nonoperative Management of Acute Epidural Hematoma Diagnosed by CT: The Neuroradiologist's Role. American Journal of Neuroradiology, 13, 853-859.

[8] Narayan, R.K., Narayan, T.M., Katz, D.A., Kornblith, P.L. and Murano, G. (1985) Lysis of Intracranial Hematomas with Urokinase in a Rabbit Model. Journal of Neurosurgery, 62, 580-586. https://doi.org/10.3171/jns.1985.62.4.0580

[9] Servadei, F., Faccani, G., Roccella, P., Seracchioli, A., Godano, U., Ghadirpour, R., et al. (1989) Asymptomatic Extradural Hematomas. Results of Multicenter Study of 158 Cases in Minor Head Injury. Acta Neurochirurgica, 96, 39-45. https://doi.org/10.1007/BF01403493

[10] Tallon, J.M., Ackroyd-Stolarz, S., Karim, S.A. and Clarke, D.B. (2008) The Epidemiology of Surgically Treated Acute Subdural and Epidural Hematomas in Patients with Head Injuries: A Population-Based Study. Canadian Journal of Surgery, 51, 339-345.

[11] Chen, T.Y., Wong, C.W., Chang, C.N., Lui, T.N., Cheng, W.C., Tsai, M.D., et al. (1993) The Expectant Treatment of "Asymptomatic" Supratentorial Epidural Hematomas. Neurosurgery, 32, 176-179. https://doi.org/10.1227/00006123-199302000-00004

[12] Bullock, R., Smith, R.M. and van Dellen, J.R. (1985) Nonoperative Management of Extradural Hematoma. Neurosurgery, 16, 602-606. https://doi.org/10.1227/00006123-198505000-00003

[13] Liu, J.T., Tyan, Y.S., Lee, Y.K. and Wang, J.T. (2006) Emergency Management of Epidural Hematoma through Burr-Hole Evacuation and Drainage. A Preliminary Report. Acta Neurochirurgica, 148, 313-317. https://doi.org/10.1007/s00701-005-0723-Z

[14] Park, J., Kim, G.J. and Hwang, S.K. (2008) Thrombolytic Evacuation of Post-Craniotomy Epidural Hematomas Using Closed Suction Drains: A Pilot Study. Acta Neurochirurgica, 150, 359-366. https://doi.org/10.1007/s00701-007-1487-4

[15] Doi, E., Moriwaki, H., Komai, N. and Iwamato, M. (1982) Stereotactic Evaluation of Intracerabral Hematomas. Neurologia Medico-Chirurgica, 22, 461-467. 
https://doi.org/10.2176/nmc.22.461

[16] Findlay, J.M., Weir, B.K., Steinke, D., Tanabe, T., Gordon, P. and Grace, M. (1988) Effect of Intrathecal Thrombolytic Therapy on Subarachnoid Clot and Chronic Vasospasm in a Primate Model of SAH. Journal of Neurosurgery, 69, 723-735. https://doi.org/10.3171/jns.1988.69.5.0723

[17] Mohadjer, M., Eggert, R., May, J. and Mayfrank, L. (1990) CT-Guided Stereotactic Fibrinolysis of Spontaneous and Hypertensive Cerebellar Hemorrhage: Long-Term Results. Journal of Neurosurgery, 73, 217-222. https://doi.org/10.3171/jns.1990.73.2.0217

[18] Itabashi, H.H., Andrews, J.M., Tomiyasu, U., Erlich, S.S. and Sathyavagiswaran, L. (2007) Forensic Neuropathology: A Practical Review of the Fundamentals: Chapter 3-Dating/Aging of Common Lesions in Neuropathology. Elsevier, Amsterdam, 4969.

[19] Pang, D., Horton, J.A., Herron, J.M., Wilberger, J.E. and Vries, J.K. (1983) Nonsurgical Management of Extradural Hematomas in Children. Journal of Neurosurgery, 59, 958-971. https://doi.org/10.3171/jns.1983.59.6.0958

[20] Kunamneni, A., Ravuri, B.D., Ellaiah, P., Prabhakhar, T. and Saisha, V. (2008) Urokinase-A Strong Plasminogen Activator. Biotechnology and Molecular Biology Reviews, 3, 58-70.

[21] Bell, W.R. (1975) Thrombolytic Therapy. A Comparison between Urokinase and Streptokinase. Seminars in Thrombosis and Hemostasis, 2, 1-13.

https://doi.org/10.1055/s-0028-1086111

[22] Urokinase Pulmonary Embolism Trial Study Group (1974) Urokinase-Streptokinase Embolism Trial. JAMA, 229, 1606-1613. https://doi.org/10.1001/jama.1974.03230500024021

[23] Pang, D., Sclabassi, R.J. and Horton, J.A. (1986) Lysis of Intraventricuar Blood Clot with Urokinase in a Canine Model: Part 3. Effective of Intraventricular Urokinase on Clot Lysis and Posthemorrhagic Hydrocephalus. Neurosurgery, 19, 553-572. https://doi.org/10.1227/00006123-198610000-00010

[24] Jamieson, K.G. and Yelland, J.D. (1968) Extradural Hematoma. Report of 167 Cases. Journal of Neurosurgery, 29, 13-23. https://doi.org/10.3171/jns.1968.29.1.0013

[25] Offner, P.J., Pham, B. and Hawkes, A. (2006) Nonoperative Management of Acute Epidural Hematomas: A “No-Brainer". The American Journal of Surgery, 192, 801805.

[26] Bezircioglu, H., Ersahin, Y., Demircivi, F., Yurt, I., Donertas, K. and Tektas, S. (1996) Nonoperative Treatment of Acute Extradural Hematomas: Analysis of 80 Cases. Journal of Trauma, 41, 696-698. https://doi.org/10.1097/00005373-199610000-00016

[27] Shah, M.V. (1999) Conservative Management of Epidural Hematomas: Is It Safe and Is It Cost-Effective? American Journal of Neuroradiology, 20, 115-116.

[28] Pozzati, E. and Tognetti, F. (1986) Spontaneous Healing of Acute Extradural Hematomas: Study of Twenty-Two Cases. Neurosurgery, 18, 696-700. https://doi.org/10.1227/00006123-198606000-00003

[29] Noguchi, M., Inamasu, J., Kawai, F., Kato, E., Kuramae, T., Oyanagi, T., et al. (2010) Ultrasound-Guided Needle Aspiration of Epidural Hematoma in a Neonate after Vacuum-Assisted Delivery. Child s Nervous System, 26, 713-716. https://doi.org/10.1007/s00381-009-1072-7

[30] Smets, K.J. and Vanhauwaert, D. (2010) Treatment of Cranial Epidural Hematoma 
in a Neonate by Needle Aspiration of a Communicating Cephalhematoma. European Journal of Pediatrics, 169, 617-619. https://doi.org/10.1007/s00431-009-1071-4

[31] Liu, W., Ma, L., Wen, L., Shen, F., Sheng, H., Zhou, B., et al. (2008) Drilling Skull plus Injection of Urokinase in the Treatment of Epidural Hematoma: A Preliminary Study. Brain Injury, 22, 199-204. https://doi.org/10.1080/02699050801895407

[32] Morgenstern, L.B., Demchuk, A.M., Kim, D.H., Frankowski, R.F. and Grotta, J.C. (2001) Rebleeding Leads to Poor Outcome in Ultra-Early Craniotomy for Intracerebral Hemorrhage. Neurology, 56, 1294-1299. https://doi.org/10.1212/WNL.56.10.1294

[33] Lofgren, J. (1986) Traumatic Intracranial Hematomas. Pathophysiological Aspects on Their Course and Treatment. Acta Neurochirurgica Supplement, 36, 151-154.

[34] Mclaurin, R.L. and Ford, L.E. (1964) Extradural Hematoma; Statistical Survey of Forty-Seven Cases. Journal of Neurosurgery, 21, 364-371. https://doi.org/10.3171/jns.1964.21.5.0364

[35] Chang, G.Y., Woo, Y.J., Seong, H.K., Oh, L.K. and Min, S.K. (2016) The Effectiveness of Subdural Drains Using Urokinase after Burr Hole Evacuation of Subacute Subdural Hematoma in Elderly Patients: A Prelimilary Report. Korean Journal of Neurotrauma, 12, 101-106. https://doi.org/10.13004/kjnt.2016.12.2.101

[36] Borovich, B., Braun, J., Guilburd, J.N., Zaaroor, M., Michich, M., Levy, L., et al. (1985) Delayed Onset of Traumatic Extradural Hematoma. Journal of Neurosurgery, 63, 30-34. https://doi.org/10.3171/jns.1985.63.1.0030

Submit or recommend next manuscript to OALib Journal and we will provide best service for you:

- Publication frequency: Monthly

- 9 subject areas of science, technology and medicine

- Fair and rigorous peer-review system

- Fast publication process

- Article promotion in various social networking sites (LinkedIn, Facebook, Twitter, etc.)

- Maximum dissemination of your research work

Submit Your Paper Online: Click Here to Submit

Or Contact service@oalib.com 